\title{
Potential of oil palm empty fruit bunch (OPEFB) and sugarcane bagasse fibers for thermal insulation application-a review
}

\begin{abstract}
In Malaysia, the majority of household electricity (45\%) is consumed by air conditioning. Such high energy consumption is mainly caused by inefficient insulating materials with poor thermal performance. Hence, the selection of a thermal wall insulator with low thermal conductivity is vital to reduce the energy consumption. Utilizing agro waste based thermal insulation materials has become an efficient and economical method for energy efficient building. In this review, the standards required for an efficient thermal insulator have been addressed. Moreover, thermal, acoustic, physical and mechanical performance of oil palm empty fruit bunch fiber (OPEFB) and sugarcane bagasse fiber and their composites are described in detail. Optimal fiber loading and different surface modifications were reported to enhance the thermal performance, acoustic, physical and mechanical performance of composites. From the observations, it has been concluded that agro waste based OPEFB and bagasse fibers can act as an efficient thermal insulator, which will significantly reduce the excess utilization of energy and thus the costs.
\end{abstract}

Keyword: Oil palm empty fruit bunch fiber; Sugarcane bagasse; Thermal insulation; Polymer; Mechanical properties; Acoustic properties 